\title{
Increased Severe Adverse Outcomes and Decreased Emergency Room Visits for Pyelonephritis: First Report of Collateral Damage during COVID-19 Pandemic in Urology
}

\author{
Hendrik Borgmann ${ }^{\mathrm{a}} \quad$ Julian P. Struck ${ }^{\mathrm{b}}$ Angelika Mattigk ${ }^{\mathrm{c}} \quad$ Mike Wenzel $^{\mathrm{d}}$ \\ Adrian Pilatz $^{\mathrm{e}}$ Jennifer Kranz ${ }^{\mathrm{f} g}$ $\quad$ Richard Weiten $^{\mathrm{h}} \quad$ Nicolas von Landenberg $^{\mathrm{i}}$ \\ Philipp Julian Spachmann ${ }^{j} \quad$ Cem Aksoy $^{k} \quad$ Axel Haferkamp $^{a}$ Katharina Boehm ${ }^{a}$ \\ aDepartment of Urology, University Medical Center, Johannes Gutenberg University, Mainz, Germany; ${ }^{b}$ Department \\ of Urology, University Hospital Luebeck, Luebeck, Germany; 'Department of Urology, University Hospital UIm,

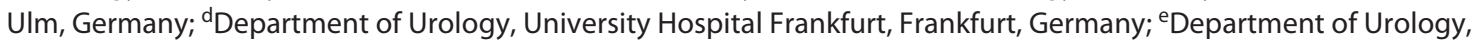 \\ Pediatric Urology and Andrology, Justus-Liebig-University Giessen, Giessen, Germany; fDepartment of Urology, \\ St.-Antonius Hospital Eschweiler, Eschweiler, Germany; ${ }^{9}$ Department of Urology and Kidney Transplantation,

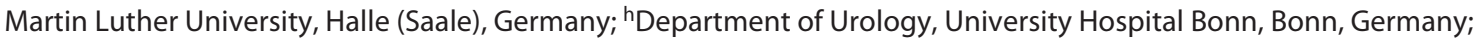 \\ 'Department of Urology, Marien Hospital Herne, Ruhr-University Bochum, Herne, Germany; 'Department of Urology,

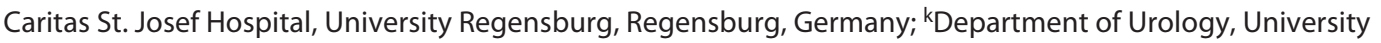 \\ Hospital Dresden, Dresden, Germany
}

\section{Keywords}

Outcome $\cdot$ Morbidity $\cdot$ Side effect $\cdot$ Coronavirus ·

SARS-CoV-2 · 2019-nCoV · Urinary infection · Pyelonephritis

\begin{abstract}
Purpose: The coronavirus disease 2019 (COVID-19) pandemic is disrupting urology health-care worldwide. Reduced emergency room visits resulting in adverse outcomes have most recently been reported in pediatrics and cardiology. We aimed to compare patients with emergency room visits for pyelonephritis in 2019 (pre-COVID-19 era) and within the first wave of pandemic in 2020 (COVID-19 era) with regard to the number of visits and severe adverse disease outcomes. Methods: We performed a retrospective multicentre study comparing characteristics and outcomes of patients with pyelonephritis, excluding patients with hydronephrosis due to stone disease, in 10 urology departments in Germany during a 1-month time frame in March and April in each 2019 and
\end{abstract}

2020. Results: The number of emergency room visits for pyelonephritis in the COVID-19 era was lower (44 patients, $37.0 \%$ ) than in the pre-COVID-19 era (76 patients, $63.0 \%$ ), reduction rate: $42.1 \%(p=0.003)$. Severe adverse disease outcome was more frequent in the COVID-19 era ( $9 / 44$ patients, $20.5 \%)$ than in the pre-COVID-19 era (5/76 patients, $6.6 \%$, $p=0.046$ ). In detail, 7 versus 3 patients needed monitoring (15.9 vs. $3.9 \%), 2$ versus no patients needed intensive-care treatment (4.5 vs. $0 \%$ ), 2 versus no patients needed drain placement ( 4.5 vs. $0 \%), 2$ versus no patients had a nephrectomy (4.5 vs. $0 \%$ ), and 2 versus 1 patient died (4.5 vs. $1.3 \%$ ). Conclusion: This report of collateral damage during $\mathrm{CO}$ VID-19 showed that emergency room visits were decreased, and severe adverse disease outcomes were increased for patients with pyelonephritis in the COVID-19 era. Health authorities should set up information campaign programs actively encouraging patients to utilize emergency room services in case of severe symptoms specifically during the actual second wave of pandemic.

(c) 2021 S. Karger AG, Basel karger@karger.com

www.karger.com/uin

Karger ${ }^{\prime}=$

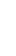

(C) 2021 S. Karger AG, Basel
Hendrik Borgmann

Department of Urology, University Medical Center

Johannes Gutenberg University

Langenbeckstr. 1, DE-55131 Mainz (Germany)

hendrik.borgmann@ unimedizin-mainz.de 


\section{Introduction}

The coronavirus disease 2019 (COVID-19) pandemic is disrupting urology health-care worldwide [1]. Surgical and oncological care in urology is prioritized and carried out according to local infection load, and resources aiming to minimize harm to non-COVID-19-related health care in urology [2,3]. To this end, the European Association of Urology has adapted their comprehensive guidelines to the COVID-19 era, with recommendations tired for 4 levels of priority: low, intermediate, high, and emergency [4]. Similarly, the German urological community revealed recommendations for the treatment of emergency patients [5]. Concerning the management of urological emergency conditions, pathways for urosepsis, urinary retention, and gross hematuria have been proposed [6].

Recent correspondences outline that patients' emergency room visits are remarkably reduced during COVID-19 time compared to similar time periods in 2019 [7]. Patients' reticence for accessing emergency health care is caused by fear of acquiring SARS-CoV-2 in the hospital environment, uncertainty of the availability of health-care services, and social responsibility for using resources in times of scarcity [8]. Concerning urological emergencies, timely emergency room visit of patients is particularly relevant for urosepsis due to its high mortality rate of $18-28 \%$ [9]. We observed in our daily clinical urology practice that patients with pyelonephritis most recently presented both in fewer numbers and with increased disease severity. Pyelonephritis is a condition that can lead to urosepsis and usually develops slowly presenting with dysuria and/or increasing flank pain and/or fever. Due to these disease characteristics, pyelonephritis qualifies as suitable indicator disease for generating the hypothesis of fewer and delayed patient visits to the emergency room leading to increased severe adverse disease outcomes. To this date, few clinical data on emergency room visits of urology patients during COVID-19 [10] and no clinical data on adverse outcomes of non-COVID-19-related health care in urology exist. To investigate this recent observation, we performed a multicentre retrospective study to compare patients with emergency room visits for pyelonephritis in 2019 (non-COVID-19 era) and 2020 (COVID-19 era) with regard to the number of visits and severe adverse disease outcomes.

\section{Patients and Methods}

\section{Study Population}

We performed a retrospective data analysis from 120 patients treated for pyelonephritis at 10 urology departments in Germany.
Patients were selected as having pyelonephritis according to the ICD code of the patient file and clinical symptoms. Patients with acute obstructive uropathy due to a ureteral stone were not included. Patients with antecedent DJ-insertion and readmission because of Pyelonephritis were defined as reflux-pyelonephritis patients.

The first group consisted of consecutive patients who searched urological help due to pyelonephritis between March 12 and April 12, 2019. This time span was defined as pre-COVID-19 era. The second group consisted of consecutive patients with a first consultation between March 12 and April 12, 2020, starting from the beginning of COVID-19 pandemic in Germany (COVID-19 era). All patients presented as emergency patients. The local Ethics Board approved this study.

\section{Measures}

Demographical information about age and gender, as well as date of consultation, date of discharge, date of first symptom presentation, previous antibiotic therapy, and prior DJ-insertion due to ureteral stones, was collected. Duration of hospitalization was computed. Additionally laboratory and clinical parameter were evaluated: C-reactive protein (CRP) $(\mathrm{mg} / \mathrm{L})$, leukocyte count (number $/ \mu \mathrm{L}$ ), and fever $\left({ }^{\circ} \mathrm{C}\right)$. We focused on 5 outcome parameters: first need of monitoring/intermediate care unit, second need of intensive care, third need of drain placement for abscess, fourth nephrectomy, and finally death. The appearance of any of these outcomes was defined as adverse outcome of pyelonephritis.

\section{Statistical Analyses}

Descriptive statistic was used for patients' characteristics. Patients were stratified according to treatment era (COVID-19 vs. pre-COVID-19 era) (Table 1) and according to outcome (adverse vs. mild outcome) (Table 2).

Stacked columns were used to depict numbers of inpatients and outpatients (Fig. 1a) as well as proportion of patients with adverse versus mild outcome of pyelonephritis (Fig. 1b), stratified according to the treatment time, COVID-19 versus the pre-COVID-19 era. Boxplots were fitted to depict distributions of leucocytes and CRP counts, stratified according to COVID-19- versus pre-COVID-19-era patients (Fig. 2). All statistical analyses were performed using RStudio v0.98.953 (R Project for Statistical Computing, www.R-project.org).

\section{Results}

Overall, 120 patients out of 10 urological departments were included. The number of emergency room visits for pyelonephritis in the COVID-19 era was lower (44 patients, $37.0 \%)$ than in the pre-COVID-19 era (76 patients, $63.0 \%)(p=0.003)$. Figure 1a depicts the resulting reduction rate of $42.1 \%$ of emergency room visits during COVID-19 era. Patients' emergency room visits per center were decreased during COVID-19 era (median 4 [IQR]) compared to pre-COVID-19 era (median 7 [IQR], $p=$ $0.0001)$. Thirty-three $(27.5 \%)$ patients were treated as outpatients (30.3 vs. $22.7 \%, p=0.5)$ and $87(72.5 \%)$ pa- 
Table 1. Patients' characteristics

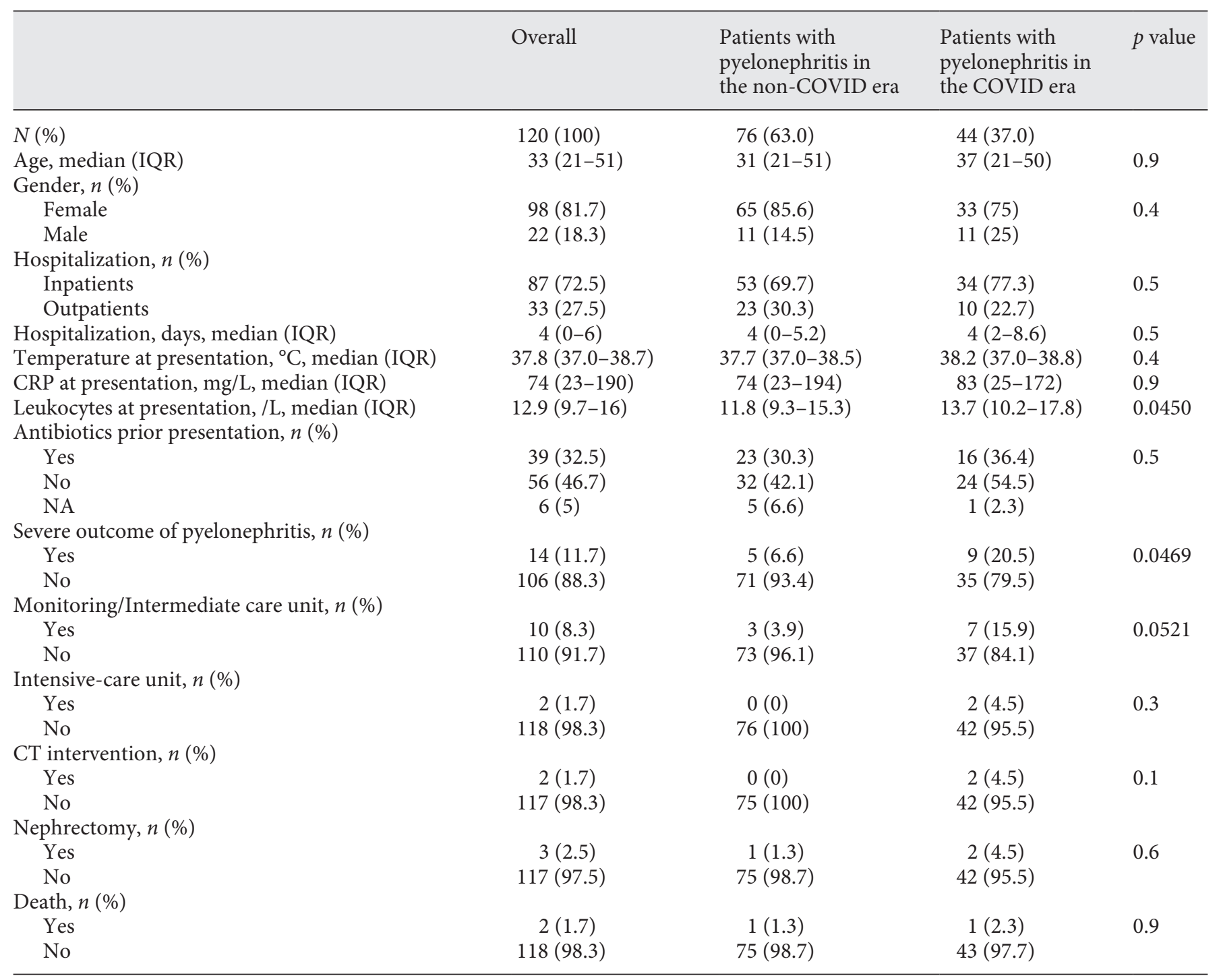

CRP, C-reactive protein; COVID-19, coronavirus disease 2019.

tients were treated as inpatients ( 69.7 vs. $77.3 \%, p=0.5$ ) during the COVID versus pre-COVID-19 era.

Median age of all patients was 31 years (IQR 21-51). Patients in the pre-COVID-19 era were 31-years (21-51) old versus 37 (21-50) in the COVID-19 era, $p=0.9$. Most patients were female ( 81.7 vs. $18.3 \%$ ) with no statistically significant difference between COVID-19 and pre-COVID-19 era ( 85.6 vs. $75.0 \%, p=0.4$ ). Median duration of hospitalization was overall 4 days (0-6) and $4(0-5.2)$ versus 4 (2-8.6), $p=0.5$ days (COVID-19 vs. pre-COVID-19 era). The median temperature of patients was $37.8^{\circ} \mathrm{C}$ (37.0-38.7) without significant difference between CO-
VID-19 versus pre-COVID-19-era patients $\left(37.7^{\circ} \mathrm{C}[37.0-\right.$ $38.5]$ versus $38.2^{\circ} \mathrm{C}$ [37.0-38.8], $p=0.4$ ). Figure 2 shows that inflammatory blood values were higher in patients treated in the COVID-19 era compared to laboratory results in patients in the pre-COVID-19 era (leukocytes 13.7 vs. $11.8 / \mu \mathrm{L}, p=0.045$ and CRP: 83 vs. $74 \mathrm{mg} / \mathrm{L}, p=0.9$ ). None of the patients had a proven COVID-19 infection.

Overall 14 patients (11.7\%) presented at least one of the above defined adverse outcome factors. Figure $1 \mathrm{~b}$ shows that adverse outcome was more frequent in patients in the COVID-19 era (9/44 patients, 20.5\%) than in the pre-COVID-19 era (5/76 patients, $6.6 \%, p=0.046)$. In 


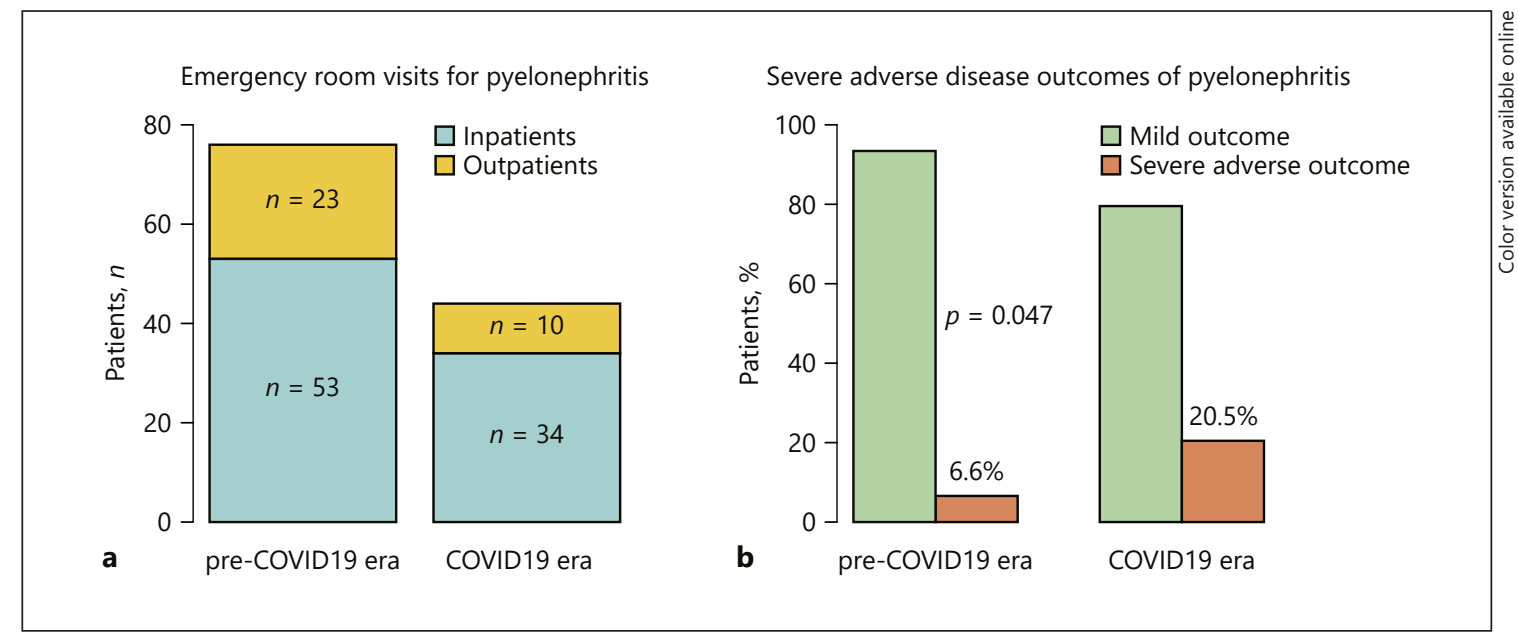

Fig. 1. a Depicts numbers of patients emergency room visits for pyelonephritis resulting in inpatient and outpatient treatments, stratified according to the treatment time, pre-COVID-19 era versus COVID-19 era. b Depicts proportions of patients with mild versus severe adverse outcomes of pyelonephritis, stratified according to the treatment time, pre-COVID-19 era versus COVID-19 era. COVID-19, coronavirus disease 2019.

Table 2. Patients' characteristics stratified according to severe and mild outcome of pyelonephritis

\begin{tabular}{|c|c|c|c|c|}
\hline & Overall & $\begin{array}{l}\text { Patients with } \\
\text { mild outcome of } \\
\text { pyelonephritis }\end{array}$ & $\begin{array}{l}\text { Patients with } \\
\text { severe outcome of } \\
\text { pyelonephritis }\end{array}$ & $p$ value \\
\hline$N(\%)$ & $120(100)$ & $106(88.3)$ & $14(11.7)$ & \\
\hline Age, years, median (IQR) & $33(21-51)$ & $32(21-51)$ & $35(21-50)$ & 0.9 \\
\hline \multicolumn{5}{|l|}{ Gender, $n(\%)$} \\
\hline Female & $98(81.7)$ & $87(82.1)$ & $11(78.6)$ & 1 \\
\hline Male & $22(18.3)$ & $19(17.9)$ & $3(21.4)$ & \\
\hline Hospitalization, days, median (IQR) & $4(0-6)$ & $4(0-5)$ & $7.5(6-11)$ & $<0.001$ \\
\hline Temperature at presentation, ${ }^{\circ} \mathrm{C}$, median (IQR) & $37.8(37.0-38.7)$ & $37.7(37.0-38.7)$ & $38.2(37.0-38.9)$ & 0.6 \\
\hline $\mathrm{CRP}$ at presentation, $\mathrm{mg} / \mathrm{L}$, median (IQR) & $74(23-190)$ & $60(22-168)$ & $237(120-380)$ & 0.001 \\
\hline Leukocytes at presentation, /L, median (IQR) & $12.9(9.7-16)$ & $12.2(9.7-15.3)$ & $17(13.8-23.3)$ & 0.01 \\
\hline \multicolumn{5}{|l|}{ Antibiotics prior presentation, $n(\%)$} \\
\hline Yes & $39(32.5)$ & $34(32.1)$ & $5(35.7)$ & 0.6 \\
\hline No & $56(46.7)$ & $50(47.2)$ & $6(42.9)$ & \\
\hline na & $6(5)$ & $6(5.7)$ & $0(0)$ & \\
\hline \multicolumn{5}{|l|}{ Prior DJ-Insertion/reflux pyelonephritis, $n$ (\%) } \\
\hline Yes & $11(9.2)$ & $11(10.4)$ & $0(0)$ & 0.09 \\
\hline No & $73(60.8)$ & $62(58.5)$ & $11(78.6)$ & \\
\hline na & $17(14.2)$ & $17(16)$ & $0(0)$ & \\
\hline \multicolumn{5}{|l|}{ Time of presentation, $n(\%)$} \\
\hline Non-COVID era & $76(63.3)$ & $71(67)$ & $5(35.7)$ & 0.047 \\
\hline COVID era & $44(36.7)$ & $35(33)$ & $9(64.3)$ & \\
\hline
\end{tabular}

CRP, C-reactive protein; COVID-19, coronavirus disease 2019.

detail, 7 versus 3 patients needed monitoring (15.9 vs. $3.9 \%), 2$ versus no patients needed intensive-care treatment ( 4.5 vs. $0 \%), 2$ versus no patients had a drain placement for abscess ( 4.5 vs. $0 \%), 2$ versus no patients had a nephrectomy ( 4.5 vs. $0 \%)$, and 2 versus 1 patient died ( 4.5 vs. $1.3 \%$ ) (Table 1 ).

Patients with adverse outcome of pyelonephritis $(14 / 120=11.7 \%)$ were not significantly older (32 vs. 35 
Fig. 2. Leukocytes count and CRP in patients with pyelonephritis stratified according to time of treatment (pre-COVID vs. COVID era). CRP, C-reactive protein. COVID-19, coronavirus disease 2019.

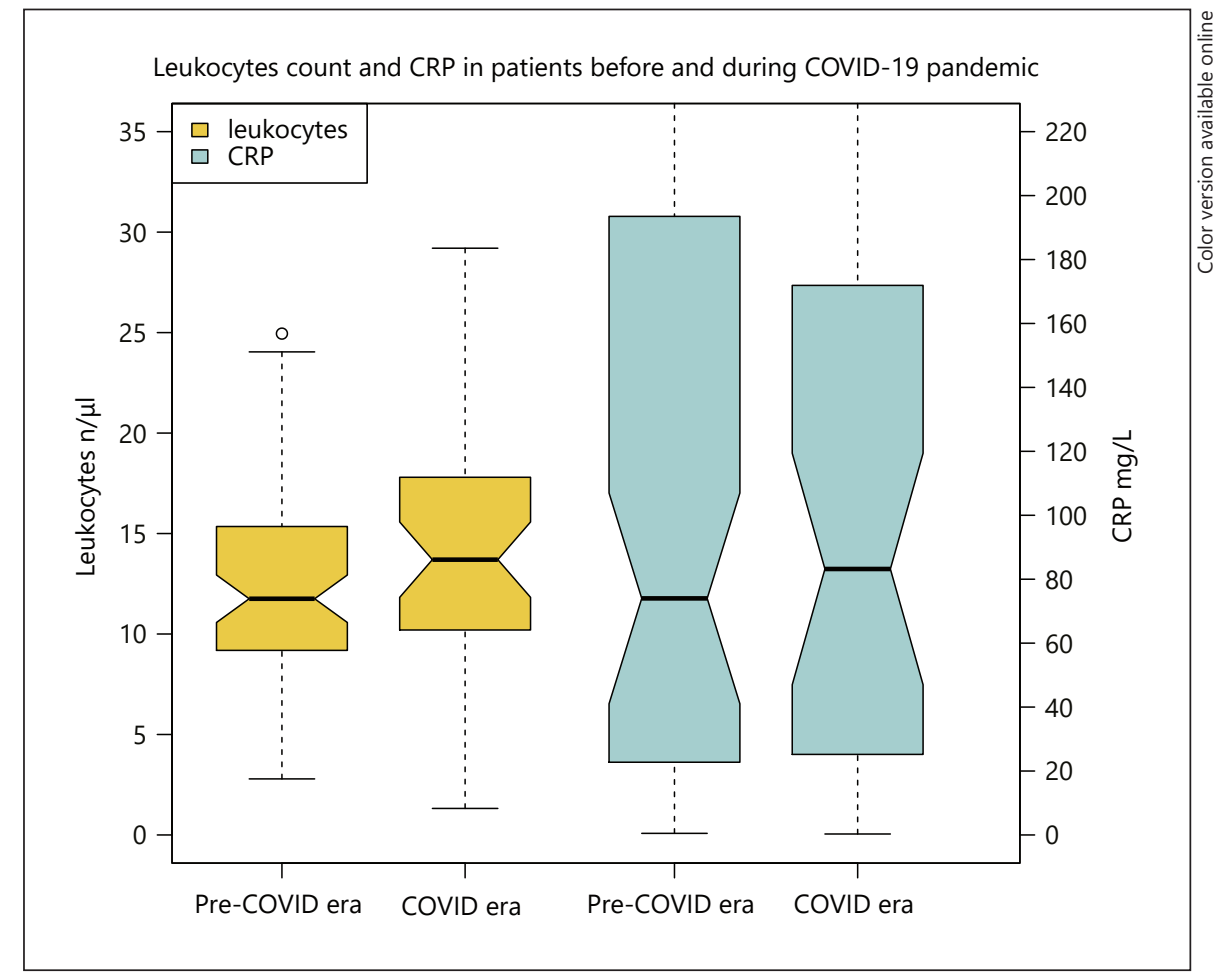

years; $p=0.9$ ), had no higher temperature at presentation ( 37.7 vs. $38.2^{\circ} \mathrm{C} ; p=0.6$ ), and did not differ in terms of gender (female 82.1 vs. 78.6\%) (Table 2). Proportion of patients with prior antibiotic therapy (32.1 vs. $35.7 \%$; $p=$ 0.6 ) or prior DJ-Insertion (10.4 vs. $0 \%$ ) was not statistically different between patients with mild versus adverse outcome of pyelonephritis. In contrast, patients with adverse outcome of pyelonephritis had a longer hospital stay ( 7.5 vs. 4 days; $p<0.001$ ), a higher CRP at presentation (237 vs. $60 \mathrm{mg} / \mathrm{L} ; p=0.001)$ and a higher leukocyte count (17 vs. $12.3 / \mu \mathrm{L}, p=0.01)$.

\section{Discussion}

In this multicentre retrospective study, we found a significant reduction of $42 \%$ of emergency room visits of patients for pyelonephritis during the COVID-19 era compared to pre-COVID-19 era. Vice versa, adverse outcomes including monitoring, intensive-care treatment, drain placement for abscess, nephrectomy, and death were more frequent in the COVID-19 era (combined adverse outcome rate: $20.5 \%)$ compared to the pre-COVID-19 era $(6.6 \%, p=0.045)$.

To our best knowledge, this study is one of the first reports of clinical data on adverse disease outcomes in urology during COVID-19 pandemic. Currently, remarkable feats are undertaken to deliver optimal urology care during COVID-19 [11]. However, until a vaccine is found [12], the burden of surgical and oncological urology care that we are facing ahead is enormous and adverse outcomes due to delay in diagnosis and treatment are most likely to occur $[13,14]$. While prioritization of urology care is directly in our hands, other collateral effects of the pandemic like delayed emergency room visits are not.

Our observation of fewer emergency room visits during COVID-19 for pyelonephritis is generally in line with data from Italy, Portugal, and China that showed a decrease ranging from 38 to $88 \%$ [ $7,8,15-18]$. In Austria, a relative reduction of $39.4 \%$ in admissions for acute coronary syndrome has been reported during COVID-19 era which was theoretically estimated to an increase of 110 deaths [19].

Although we did not directly prove delayed access to emergency care in our cohort of patients, we assume a link between decreased emergency room visits and increased severe adverse outcomes during the COVID-19 era. Since pyelonephritis is a disease that can develop and deteriorate slowly over a period of days, we assume that patients in the COVID-19 era presented to the emergency room with significant delay, which might have led to 
increased adverse outcomes. In fact, leukocyte counts were significantly higher in our study patients in the COVID-19 era than in the non-COVID-19 era. The symptom of fever in patients with pyelonephritis is also a cardinal symptom of SARS-CoV-2 infection. Indeed, patients with fever and respiratory symptoms were in Germany advised as a first action to stay at home and contact the local health authority by phone for further instructions and triage. On the other hand, we do not assume that care delivery in the German urology departments has negatively impacted patient outcomes since the health-care system in Germany has not experienced capacity pressure thus far.

Consequently, actively encouraging patients to visit the emergency room in case of a real emergency during COVID-19 era is the call-to-action derived from our study, especially in times where infection rate is rising again. Patients' barrier-free access to early telemedicine video-consultation might be another option to optimize triage and ensure timely emergency room visits [20].

Our study has important strengths. Most importantly, we report important clinical data of adverse disease outcomes in urology during COVID-19 pandemic. In this regard, timely adverse outcome reporting of collateral damages during the pandemic is of utmost importance since adjustments in clinical care can be made in time to reduce collateral damage and maintain optimal patient outcomes. Moreover, the multicentre study design reflects a true representation of patients with pyelonephritis in Germany, a country severely affected by COVID-19. Finally, clinically relevant severe adverse outcomes were measured as outcome parameter and were more frequent in the COVID-19 era. Limitations include those inherit to the retrospective study design. Furthermore, patient flow and access to emergency room might differ between health-care systems.

\section{Conclusion}

Emergency room visits were decreased, and severe adverse disease outcomes were increased for patients with pyelonephritis in the COVID-19 era. Health authorities should set up information campaign programs actively encouraging patients to utilize emergency room services in case of severe symptoms.

\section{Acknowledgement}

The authors thank Naomi Mohr for reflection and input to this study design.

\section{Statement of Ethics}

Ethical approval was waived by the local Ethics Committee of University Mainz in view of the retrospective nature of the study and all the procedures being performed were part of the routine care. Therefore, we did not get an approval reference number.

\section{Conflict of Interest Statement}

The authors have no potential conflicts of interest.

\section{Funding Sources}

The authors did not receive any funding.

\section{Author Contributions}

H. Borgmann: project development, data collection or management, data analysis, and manuscript writing/editing. J.P. Struck: data collection or management and manuscript writing/editing. A. Mattigk: data collection or management and manuscript writing/editing. M. Wenzel: data collection or management and manuscript writing/ editing. A. Pilatz: data collection or management and manuscript writing/editing. J. Kranz: data collection or management and manuscript writing/editing. R. Weiten: data collection or management and manuscript writing/editing. N. von Landenberg: data collection or management and manuscript writing/editing. P.J. Spachmann: data collection or management and manuscript writing/editing. C. Aksoy: data collection or management and manuscript writing/editing. A. Haferkamp: data collection or management and manuscript writing/editing. K. Boehm: project development, data collection or management, data analysis, and manuscript writing/editing. All authors read and approved the final version of the manuscript.

\section{References}

1 Puliatti S, Eissa A, Eissa R, Amato M, Mazzone E, Dell'Oglio P, et al. COVID-19 and urology: a comprehensive review of the literature. BJU Int. 2020;125(6):E7-14.
2 Stensland KD, Morgan TM, Moinzadeh A, Lee CT, Briganti A, Catto JWF, et al. Considerations in the triage of urologic surgeries during the COVID-19 pandemic. Eur Urol. 2020;77(6):663-6.
3 Gillessen S, Powles T. Advice regarding systemic therapy in patients with urological cancers during the COVID-19 pandemic. Eur Urol. 2020;77(6):667-8. 
4 Ribal MJ, Cornford P, Briganti A, Knoll T, Gravas S, Babjuk M, et al. European association of urology guidelines office rapid reaction group: an organisation-wide collaborative effort to adapt the european association of urology guidelines recommendations to the coronavirus disease 2019 era. Eur Urol. 2020;78(1):21-28.

5 Kriegmair MC, Kowalewski KF, Lange B, Heininger A, Speck T, Haas H, et al. [Urology in the corona-virus pandemic: a guideline 4/20]. Urologe A. 2020;59(4):442-9.

6 Simonato A, Giannarini G, Abrate A, Bartoletti R, Crestani A, De Nunzio C, et al. Pathways for urology patients during the COVID-19 pandemic. Minerva Urol Nefrol. 2020;72(3):376-83.

7 Motterle G, Morlacco A, Iafrate M, Bianco M, Federa G, Xhafka O, et al. The impact of COVID-19 pandemic on urological emergencies: a single-center experience. World J Urol. 2020:1-5.

8 Lazzerini M, Barbi E, Apicella A, Marchetti F, Cardinale F, Trobia G. Delayed access or provision of care in Italy resulting from fear of COVID-19. Lancet Child Adolesc Health. 2020;4(5):e10-11.
9 Martin GS, Mannino DM, Eaton S, Moss M. The epidemiology of sepsis in the United States from 1979 through 2000. N Engl J Med. 2003;348(16):1546-54.

10 Porreca A, Colicchia M, D'Agostino D, Amenta M, Corsaro A, Zaramella S, et al. Urology in the time of coronavirus: reduced access to urgent and emergent urological care during the coronavirus disease 2019 outbreak in Italy. Urol Int. 2020;104(7-8):631-6.

11 Ahmed K, Hayat S, Dasgupta P. Global challenges to urology practice during COVID-19 pandemic. BJU Int. 2020;125(6):E5-6.

12 Desouky E. BCG versus COVID-19: impact on urology. World J Urol. 2020:1-5.

13 Campi R, Amparore D, Capitanio U, Checcucci E, Salonia A, Fiori C, et al. Assessing the burden of nondeferrable major uro-oncologic surgery to guide prioritisation strategies during the COVID-19 pandemic: insights from three Italian high-volume referral centres. Eur Urol. 2020;78(1):11-15.

14 CJD W. Risks from deferring treatment for genitourinary cancers: a collaborative review to Aid Triage and management during the COVID-19 pandemic. Eur Urol. 2020;78(1): $29-42$.
15 Guo H, Zhou Y, Liu X, Tan J. The impact of the COVID-19 epidemic on the utilization of emergency dental services. J Dent Sci. 2020.

16 Tartari F, Guglielmo A, Fuligni F, Pileri A. Changes in emergency service access after spread of COVID19 across Italy. J Eur Acad Dermatol Venereol. 2020;34(8):e350-e1.

17 Madanelo M, Ferreira C, Nunes-Carneiro D, Pinto A, Rocha MA, Correia J, et al. The impact of the COVID-19 pandemic on the utilization of emergency urological services. BJU Int. 2020;126(2):256-8.

18 Porreca A, Colicchia M, D'Agostino D, Amenta M, Corsaro A, Zaramella S, et al. Urology in the time of coronavirus: reduced access to urgent and emergent urological care during the coronavirus disease 2019 outbreak in Italy. Urol Int. 2020;104(7-8):631-6.

19 Metzler B, Siostrzonek P, Binder RK, Bauer A, Reinstadler SJ. Decline of acute coronary syndrome admissions in Austria since the outbreak of COVID-19: the pandemic response causes cardiac collateral damage. Eur Heart J. 2020;41(19):1852-3.

20 Connor MJ, Winkler M, Miah S. COVID-19 pandemic: is virtual urology clinic the answer to keeping the cancer pathway moving? BJU Int. 2020;125(6):E3-4. 